\title{
REPRESENTATION OF BOOLEAN HYPOLATTICES
}

\author{
JOHN BORIS MILLER
}

\begin{abstract}
The principal result is a representation theorem for relatively distributive, relatively complemented hypolattices with zero, generalizing the Stone representation theorem for a Boolean lattice. It uses the small product of a family of Boolean lattices which are maximal sublattices of the hypolattice. The paper also characterizes the maximal sublattices when the hypolattice is coherent; and it gives several examples of hypolattices, including hypolattices of subgroups and of ideals by direct sum, and examples from relative convexity, relative closure, and cofinality.
\end{abstract}

\section{Introduction}

A hypolattice $(X, \leq)$ is a nonempty set $X$ partially ordered by $\leq$, such that for every pair $k, l \in X$ with $k<l$ the closed interval

$$
[k, \tau]=\{x \in X: k \leq x \leq l\}
$$

is a lattice, and moreover the lattice operations coincide on overlapping intervals. Writing meet and join as $\wedge$ and $\vee$ respectively, it follows that when $x, y \in[k, z]$ then $x \wedge y$ is the infimum of $x, y$ in $x$, and dually. Thus an equivalent definition of a hypolattice is that it is a poset $(X, \leq)$ in which every closed interval is a sublattice.

The definition says that for $x, y \in X$, a sufficient condition for the existence of $\inf \{x, y\}$ in $X$ is that $x, y$ have a common lower bound and a common upper bound. Thus if $x, y$ have a common lower bound

Received 29 June 1981. I am grateful to Leigh Samphier for saving me from several errors and suggesting some improvements in this paper. 
but no common upper bound, $\inf \{x, y\}$ may not exist in $X$ : the concept of hypolattice is distinct from that of conditional lattice. But $\inf \{x, y\}^{*}$ may exist in this case; so we can distinguish two types of infima: those (called de jure) which exist by virtue of the definition of a hypolattice and those (called de facto) which exist although the definition does not assert their existence. The same remarks apply to suprema.

A poset $(X, \leq)$ all of whose closed subintervals are lattices, though perhaps not sublattices, is called a weak hypolattice. Here inf $\{x, y\}$, if $x, y$ have common lower and upper bounds, may depend upon the interval in which it is taken, and likewise for $\sup \{x, y\}$.

Hypolattices arise naturally in describing the direct-sum relation between convex $Z$-subgroups of an arbitrary $Z$-group. This was shown in [3], which gave examples showing the existence of nontrivial de facto meets. We reformulate this example in a quite general group-theoretic setting in $\$ 3$ below, and give some other instances of hypolattices and weak hypolattices, for example from convexity and cofinality.

The direct-sum hypolattices are relatively distributive and relatively complemented, so it is natural to ask if the Stone representation theory for Boolean algebras can be used to obtain a representation theorem for them. We give such a representation in $\S 5$, as our main result. A final section $\$ 6$ deals with the notions of mutuality and coherence, and the existence of maximal mutual subsets.

\section{Further definitions and elementary facts}

Let $(X, \leq)$ be a hypolattice. If $X$ has a zero (that is, least element, written 0 ) then $x \vee y$, if it exists, is necessarily de jure. Most hypolattices considered in this paper have a zero.

The partially-defined operations $\wedge, \vee$ are both associative, that is,

$$
(x \wedge y) \wedge z=x \wedge(y \wedge z)=\wedge\{x, y, z\}
$$

whenever $x \wedge y,(x \wedge y) \wedge z, y \wedge z, x \wedge(y \wedge z)$ exist in $X$; and dually for $\vee$. When ( 1 ) holds the common element is written $x \wedge y \wedge z$.

The hypolattice $(X, \leq)$ is called distributive if the equation

$$
x \wedge(y \vee z)=(x \wedge y) \vee(x \wedge z)
$$


holds whenever the 3 meets and 2 joins which appear in it exist, and dually. $(X, \leq)$ is called relatively distributive if every closed interval is a distributive lattice; this is equivalent to saying that (2) holds provided the meets and joins which appear in it exist de jure, and dually. Distributivity implies relative distributivity. It is possible for $X$ to be distributive and yet for (2) to fail, for example for one side to exist but not the other. $(X, \leq)$ is called relatively complemented if every interval is a complemented lattice.

A nonempty subset $A$ of a poset $(X, \leq)$ is called convex in $X$ if $u, v \in A, u<v$ imply $[u, v] \subseteq A$. Any convex subset of a hypolattice is itself a hypolattice with respect to the relativized order.

A nonempty subset $A$ of a hypolattice $(X, \leq)$ is called a subhypolattice if $(A, \leq \mid A)$ is a hypolattice, and for all $x, y \in A$, if inf $\{x, y\}$ exists in $A$ then $x \wedge y$ exists in $X$ and the two are equal; and dually. Note that this definition does not require $x \wedge y$ to exist in $A$ if it exists in $X$. Thus it is possible to have in a lattice $X$ a subhypolattice which is not a sublattice. A sublattice of a hypolattice is a subhypolattice which is a lattice. Equivalently, a nonempty subset $A$ of a hypolattice $X$ is a sublattice of $X$ if $x, y \in A$ implies $x \wedge y$, $x \vee y$ exist in $X$ and belong to $A$. As an application of Zorn's lemma we have

PROPOSITION 2.1. In any hypolattice $(X, \leq)$, every sublattice is contained in a maximal sublattice; every maximal sublattice is convex, and contains 0 if $X$ has zero 0 .

A (hypolattice) homomorphism from a hypolattice $(X, \leq)$ to a poset $(Y, \leq)$ is a map $\phi: X+Y$ such that, all $a, b \in X$, if $a \wedge b$ exists in $X$ then $\phi(a) \wedge \phi(b)$ exists in $Y$ and $\phi(a \wedge b)=\phi(a) \wedge \phi(b)$; and dually. A (hypolattice) isomorphism from $X$ to $Y$ is one-to-one onto hypolattice homomorphism $\phi$ such that $a \wedge b$ exists in $X$ if and only if $\phi(a) \wedge \phi(b)$ exists in $Y$, and dually. Every homomorphism is an orderpreserving map. Clearly $\phi$ is an isomorphism if and only if $\phi$ and $\phi^{-1}$ are homomorphisms. Any map $f: X+y$ is called isotone when $a \leq b$ if and only if $f(a) \leq f(b)$. An ideal in $X$ is a subset $I$ such that $y \leq x \in I$ implies $y \in I$, and if $a, b \in X$ and $a \vee b$ exists then $a \vee b \in I$. 
In seeking to describe a hypolattice $(X, S)$ it is natural to look at subsets in which $\wedge, \vee$ are everywhere-defined operations. To this end, for $x, y \in X$ write

$$
x \sim y
$$

and call $x, y$ mutual when they are contained in an interval, or equivalently, when $x \wedge y, x \vee y$ exist (de jure). The relation - is reflexive and symmetric, but not in general transitive. When $X$ has a 0 or 1 then - is transitive if and only if $X$ is a lattice. The following property, when present, is to some extent a compensation for lack of transitivity. We say $(X, \leq)$ is coherent if:

for all $x, y, z$ in $x$, if $x-y, y \smile z$ and $z-x$ then $x \wedge y \wedge z$ and $x \vee y \vee z$ exist.

If $(X, \leq)$ has a zero, then to prove that it is coherent it suffices to verify the existence of $x \vee y \vee z$. Induction proves:

PROPOSITION 2.2. Let $(X, \leq)$ be a coherent hypolattice and let $x_{1}, x_{2}, \ldots, x_{n}$ be elements of $X$. If $x_{i}-x_{j}$ for alz $i, j$ in $\{1,2, \ldots, n\}$ then $x_{1} \wedge x_{2} \wedge \ldots \wedge x_{n}$ and $x_{1} \vee x_{2} \vee \ldots \vee x_{n}$ exist.

\section{Examples}

3.1 .

Let $(L, \leq)$ be any lattice and $X$ any convex subset of $L$. Then $(X, \leq \mid X)$ is a hypolattice.

Not every hypolattice can be regarded as a convex subset of a lattice. Consider for example the poset of four elements $X=\{a, b, c, d\}$ with $a<c, a<d, b<c, b<d, a\|b, c\| d$. The example can be made more elaborate by attaching disjoint lattices at $a, b$ with $a$ and $b$ as ones, and disjoint lattices at $c, d$ with $c$ and $d$ as zeros. The poset $y=X u\{e\}$ with $c<e$ and $d<e$ is an example of a weak hypolattice. It would be interesting to have a characterization of those hypolattices which are embeddable as a convex subset of a lattice.

Let $\left(L_{i}, \leq_{i}\right), i \in I$, be a family of lattices, pairwise disjoint. On $H=\bigcup_{i \in I} L_{i}$ define $x \leq y$ to mean $x, y \in L_{i}$ and $x \leq_{i} y$, for some 
$i \in I$. Then $(H, \leq)$ is a hypolattice.

\subsection{HYPOLATTICES OF DIRECT SUMMANDS}

Let $G$ be any group, let $\operatorname{Gr}(G)$ (more briefly, $\mathrm{Gr}$ ) denote the set of all subgroups of $G$. It is known that $G r$ ordered by $\subseteq$ is a lattice, possibly nonmodular, the lattice operations being given by

$$
H \wedge K=H \cap K, \quad H \vee K=\operatorname{gr}(H, K)
$$

(gr denotes the generated subgroup). Let $\underline{\underline{C}}$ be any sublattice of $(\mathrm{Gr}, \subseteq)$ which contains $(0)$ and is distributive, and let $\mathrm{E}$ be any ideal of $\underline{\underline{\mathrm{C}}}$. Define a relation $\leq_{d}$ on $\underline{\underline{\mathrm{C}}}$ as follows: for $H, M \in \underline{\underline{\mathrm{C}}}$,

$$
H \leq_{d} M \text { means } M=H \oplus K \text { for some } K \in \underline{\underline{\mathrm{E}}} \text {. }
$$

Here $H \oplus K$ denotes the usual direct sum, that is, $H \oplus K=H \vee K$ with $H \wedge K=(0)$ and $h k=k h$ for all $h \in H, k \in K$. Because $\underline{\underline{C}}$ is distributive, $K$ in (1) will be uniquely determined. We have

PROPOSITION 3.2.1. (․․ $\left.\leq_{d}\right)$ is a coherent, relatively distributive hypolattice. It has a zero if and only if $\underline{\underline{E}}=\underline{\underline{\mathrm{C}}}$, and in this case the hypolatice is relatively complemented.

The proof that $\left(\underline{\mathrm{C}}, \leq_{d}\right)$ is a hypolattice is like that in [3], Proposition $10^{\circ}$. Note that every interval $[(0), B]_{d}$ for $B \in \underline{\underline{C}}$ is relatively complemented, but an interval $[A, B]_{d}$ where $(0) \neq A<_{d} B$ need not be so. We write $[A, B]_{d}$ for an interval in $\left(\underline{\mathrm{c}}, \leq_{d}\right)$; meet and join are shown as $\wedge_{d}$, $v_{d}$. We also have the following generalization of a remark in [3].

PROPOSITION 3.2.2. Let $G$ be commutative. Let $H_{1}, H_{2} \in \underline{\underline{\mathrm{C}}} \cdot A$ necessary and sufficient condition for $H_{1} v_{d} H_{2}$ to exist is that there exist $F_{1}, F_{2}$ in $\underline{E}$ such that

$$
H_{1}=\left(H_{1} \wedge H_{2}\right) \oplus F_{1}, H_{2}=\left(H_{1} \wedge H_{2}\right) \oplus F_{2} .
$$

If such $F_{1}, F_{2}$ exist then

(3) $H_{1} \vee_{d} H_{2}=H_{1} \vee H_{2}=\left(H_{1} \wedge H_{2}\right) \oplus E_{1} \oplus E_{2}, H_{1} \wedge H_{2}=H_{1} \wedge H_{2}$. 
If $H_{1} \wedge_{d} H_{2}$ exists as a de facto meet, then it does not equal $\mathrm{H}_{1} \wedge \mathrm{H}_{2}$.

It should be emphasised that $H_{1} v_{d} H_{2}$ is in general distinct from $H_{1} \oplus H_{2}$; the first may exist without the second. If the direct sum exists, and $H_{1}, H_{2}$ both belong to $\underline{\underline{E}}$, then the direct join exists and the two are equal. If both sum and join exist but not both of $H_{1}, H_{2}$ are in $\underline{E}$ then $H_{1} v_{d} H_{2} \neq H_{1} \oplus H_{2}$.

A special case of these two propositions is discussed in [3]. There $G$ is an $Z$-group, and

$$
\underline{\underline{\mathrm{E}}}=\underline{\underline{\mathrm{C}}}=\mathrm{Cls}(G),
$$

the complete distributive lattice of all convex $Z$-subgroups of $G$, so that $H \leq{ }_{d} M$ says that $H$ is a cardinal summand of $M .\left(\mathrm{Cls}, \leq_{d}\right)$ is a relatively complemented, relatively distributive hypolattice. The subset LcCls consisting of all lattice-closed members of $\mathrm{Cls}$ is, with respect to $\leq_{d}$ relativized, a hypolattice with those same properties. In general LcCls is not a sublattice of $(\mathrm{Cls}, \subseteq)$; on the other hand it was shown in [3], $11^{\circ}$, that $\mathrm{LCCls}$ is an ideal of the hypolattice $\left(\mathrm{Cls}, \leq_{d}\right)$ when $G$ is complete, and the lattice-closure map $\mathrm{Cls} \rightarrow \mathrm{LcCls}$ is strictly order-preserving and onto, preserving all de jure meets and joins. However when $G$ is not complete, $v_{d}$ for $\mathrm{Cls}$ and LcCls may differ. These properties of LcCls are not instances of Proposition 3.2.1. Examples of de facto meets are given in [3], together with various counterexamples. By Proposition 3.2.1, $\left(\mathrm{Cls}, \leq_{d}\right)$ is coherent; it can be shown that (LcCls, $\left.\leq_{d}\right)$ is coherent if $G$ is complete.

In Proposition 3.2.2, commutativity is specified in order to be able to deduce the existence of $F_{1} \oplus F_{2}$ from $F_{1} \cap F_{2}=(0)$. Without commutativity one still has $\left(H_{1} \wedge H_{2}\right) \oplus\left(F_{1} \vee F_{2}\right)$ as the third term of (3). Commutativity is not required in Proposition 3.2.2 in the case when $G$ is an $Z$-group.

\subsection{DIRECT SUMMANDS IN RINGS}

Let $R$ be any associative ring and let $\operatorname{Id}(R)$ be the set of all two- 
sided ideals of $R, \operatorname{Gr}(R)$ the set of all subgroups of the additive group of $R .(\operatorname{Id}(R), \subseteq)$ and $(\mathrm{Gr}(R), \subseteq)$ are both complete modular lattices, and $I d$ is a closed sublattice of $\mathrm{Gr}$. The sum of two ideals is direct if and only if it is direct as a sum of subgroups. Choose any distributive sublattice $\underline{\underline{C}}$ of Id containing $(0)$, and any ideal $\underline{\underline{E}}$ of $\underline{\underline{C}}$, and define $H \leq_{d} M$ for $H, M \in \underline{\underline{C}}$ as in (1); again $\left(\underline{\mathrm{C}}, \leq_{d}\right)$ is a coherent, relatively distributive hypolattice; it has a zero and is relatively complemented if and only if $\underline{\underline{E}}=\underline{\underline{C}}$.

Leigh Samphier (Private Communication) has shown how hypolatticies occur in direct-sum-admitting categories, in generalization of 3.2 and 3.3 .

\subsection{EXAMPLES FROM CONVEXITY}

Let $V$ be any real vector space, and for nonempty subsets $A, B$ of $\checkmark$ write

$$
A \stackrel{\longrightarrow}{=} B
$$

to mean that $A$ is a subset of $B$ convex relative to $B$, that is, $A \subseteq B$ and if $y=\lambda x_{1}+(1-\lambda) x_{2}, 0<\lambda<1, x_{1}, x_{2} \in A, y \in B$ then $y \in A$. If $\mathrm{P}(V)$ denotes the power set of $V$, then

$$
\underline{\underline{S}}=(P(V) \backslash\{\varnothing\}, \underset{=}{\underline{C}})
$$

is a weak hypolattice. The infimum of any two elements $A, B$ in any interval $[K, L]$ of $\underline{\underline{\mathrm{S}}}$ is their intersection and so is independent of the interval; the supremum is $L^{\prime} \cap \operatorname{conv}(A \cup B$ ) (where conv denotes convex hull in $V$ ) which depends upon $L$ but not on $K$. It is easy to construct an example of a de facto meet in $\underline{\underline{S}}$ which equals the intersection of the two sets concerned; $c f$. $\mathrm{Cls}$, where this is not possible.

Let $O, T$ be chosen nonempty subsets of $V$, the obstacle set and base set respectively, and write $\underline{\underline{X}}$ for the collection of all subsets of $V$ which are convex relative to $V$ and which meet $T$ but not $O$. Then

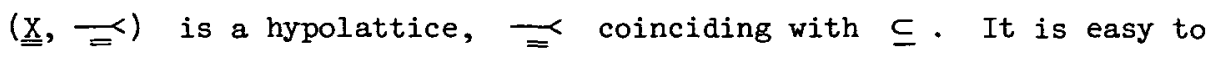
construct an example of sets $A, B, C$ in $\underline{\underline{x}}$ for which $A \vee B, B \vee C$, $C \vee A$ exist de facto in $(\underline{\underline{x}}, \underset{)}{=}$ but $A \vee B \vee C$ does not exist.

Modify this example by omitting the reference to $T$ and adjoining $\varnothing$ to $\underline{\underline{X}}$ at a least element. The resulting hypolattice is not coherent. 
3.5 .

The weak hypolattice $\underline{\underline{S}}$ described above is a particular case of the following general construction. Let $(X, \leq)$ be any lattice and $f$ any closure operator on $X$. For $a, b \in X$ write $a \leq_{f} b$ to mean $f(a) \wedge b=a$. Then $\left(x, \leq_{f}\right)$ is a weak hypolattice, in which every de jure meet $x \wedge_{f} y$ coincides with $x \wedge y$ and so is independent of the containing interval (but $\left(X, \wedge_{f}\right)$ need not be a semilattice), while for $x, y \in[k, l]$ we have $x \vee_{f} y=f(x \vee y) \wedge z$. For $\underline{\underline{\mathrm{s}}}$ the function $f$ is conv. Another example is relative closure in a topological space, $f$ be closure.

\subsection{COF INAL ITY}

The concept of cofinality gives rise to weak hypolattices in a natural way. Let $U$ be a given nonempty set. Suppose we have a collection of nonempty subsets of $U$,

$$
\mathrm{R}=\left\{A_{i}: i \in I\right\} \text {, }
$$

each $A_{i}$ equipped with some partial order $s_{i}$. These orders are not required to coincide on intersections. It suffices to assume that $R$ is $\mathrm{P}(U) \backslash\{\emptyset\}$ or more generally is any subhypolattice with respect to $\subseteq$. For each $i \in I, \underline{A}_{i}$ denotes the poset $\left(A_{i}, \leq_{i}\right)$. Write

$$
\stackrel{A_{i}}{=} \leq_{r} \stackrel{A_{j}}{\Rightarrow}
$$

to mean $A_{i} \subseteq A_{j} ;$ the restriction of $\leq_{j}$ to $A_{i}$ is $\leq_{i} ; A_{i}$ is cofinal in $\left(A_{j}, \leq_{j}\right)$. Then $\left(R, \leq_{p}\right)$ is a weak hypolattice. A de jure meet $\underline{A} \wedge_{r} \underline{B}$ has set $A \cap B$ and its order is the relativized order of $A$ or $B$, so $\wedge_{r}$ is independent of the containing interval. However $v_{r}$ may depend upon the interval.

3.7 .

Abbott [1] introduced the concept of a semi-Boolean algebra. It is defined to be a $\wedge$-semilattice with zero 0 , in which every interval $[0, a]$ is a Boolean algebra with respect to the relativized order. Every 
semi-Boolean algebra is a Boolean hypolattice (see $\$ 5$ below). For an example of a Boolean hypolattice which is not a semi-Boolean algebra see [3], p. 185.

3.8 .

A Rickart ring is a ring $R$ with identity in which 0 is the only nilpotent, and moreover for every $x \in R$ there exists an idempotent $x^{\circ}$ such that for all $y \in R, x y=0$ if and only if $y=x y y$. $F$ is partially ordered by writing $x \leq y$ to mean $x y=x^{2}$. A result of Janowitz [2] shows that $(R, \leq)$ is a Boolean hypolattice.

\section{Products of Boolean lattices}

For the representation to be given in $\$ 5$ we need to use a product of Boolean algebras; we shall define it and describe its stone space before proceeding. See Sikorski [4], pp. 50, 51 .

Let $\underline{\underline{M}}=\left(M_{i}\right)_{i \in I}$ be an arbitrary family of Boolean lattices (that is, distributive, relatively complemented lattices with zero, and having at least two elements); the relations on $M_{i}$ are written $\leq, \wedge, \vee, 0$, and $M_{i}$ is not assumed to have a one. If $M_{i}$ has a one its presence is signalled by "there exists $I \in M_{i}$ " (thus $0, I$ may be used to denote distinct elements on successive occurrences). For the theory of Boolean lattices in this generality see the original papers by Stone [5], [6].

For each $i \in I$ let $\Omega_{i}$ denote the Stone space of $M_{i}$, its elements $\underline{\underline{p}}, \underline{\underline{q}}, \ldots$ being the prime ideals of $M_{i}$, and let $\mathrm{S}_{i}$ be the stone topology; $\left(\Omega_{i}, S_{i}\right)$ is a locally compact, 0-dimensional Hausdorff space, compact if and only if there exists $1 \in M_{i}$. For any $x \in M_{i}$ and any ideal $J$ of $M_{i}$ write

$$
N(x)=\left\{\underline{\underline{p}} \in \Omega_{i}: x \notin \underline{\underline{p}}\right\}, N(J)=\left\{\underline{\underline{p}} \in \Omega_{i}: J \notin \underline{\underline{p}}\right\} .
$$

Then $\mathrm{S}_{i}$ is the collection of all sets of the form $N(J)$, and the sets $N(x)$ form a base for $\mathrm{S}_{i}$. Next write 


$$
K=\prod_{i \in I}^{*} M_{i}
$$

for the small product of the family $\underline{M}$; its elements are all tuples $x=\left(x_{i}\right)_{i \in I}, x_{i} \in M_{i}$, whose support

$$
\operatorname{supp}(x)=\left\{i \in I: x_{i} \neq 0\right\}
$$

is finite. $K$ is given the weak componentwise order: $x \leqslant y$ means $x_{i} \leq y_{i}$ in $M_{i}$ for each $i \in I$. It is routine to verify that $(K, \leqslant)$ is a Boolean lattice, the meet, join, zero 0 and relative complementation being componentwise. $K$ has a one if and only if $I$ is finite and there exists $I \in M_{i}$ for each $i \in I$. We find

THEOREM 4.1. The Stone space $\Psi$ of $K$ is homeomorphic to the topological free union of the Stone spaces $\Omega_{i}$ of the Boolean lattices $M_{i}$

By the theorem, we can express $\Psi$ itself as a topological free union, writing

$$
\Psi=\bigcup_{i \in I} \Theta_{i}
$$

the spaces $\theta_{i}$ being pairwise disjoint, clopen in $\Psi$, with $\theta_{i}$ homeomorphic to $\Omega_{i}$. In fact, for any prime ideal $P$ of $K$ write

$$
\sigma_{i}(P)=\left\{a \in M_{i}: a=x_{i} \text { for some } x \in P\right\} \text {. }
$$

Then

$$
\theta_{i}=\left\{P \in \Psi: \sigma_{i}(P) \neq M_{i}\right\}
$$

and the required homeomorphism from $\theta_{i}$ to $\Omega_{i}$ is $\sigma_{i}$.

\section{Representation of Boolean hypolattices}

Throughout this section $(X, \leq)$ denotes a Boolean hypolattice, that is, a relatively distributive, relatively complemented hypolattice with zero 0 .

$X$ has at least one maximal sublattice, by Proposition 2.1 . Let 
$\underline{\underline{M}}=\left(M_{i}\right)_{i \in I}$ be any nonempty family of maximal sublattices; none of these can be trivial. We suppose $\underline{\underline{M}}$ chosen and fixed, with $M_{i} \neq M_{j}$ when $i \neq j$; but $\underline{\underline{M}}$ need not contain every maximal sublattice, nor do we assume yet that $X=U \underline{\underline{M}}$.

Since each $M_{i}$ contains 0 and is convex, if $k, l \in M_{i}$ and $k<y<\tau$ then the relative complement of $y$ in $[k, Z]$ lies in $M_{i}$; thus $\left(M_{i}, \leq \mid M_{i}\right)$ is a Boolean lattice.

For each $x \in X$ and $i \in I$ let

$$
\theta_{i}(x)=[0, x] \cap M_{i} ;
$$

we have $\theta_{i}(x)=M_{i}$ if and only if there exists $1 \in M_{i}$ and $x=1$; if there does not exist $1 \in M_{i}$ then $\theta_{i}(x)$ is a proper ideal of $M_{i}$, and hence

$$
\lambda_{i}(x)=N\left(\theta_{i}(x)\right)=\left\{\underline{\underline{p}} \in \Omega_{i}:[0, x] \cap M_{i} \notin \underline{\underline{p}}\right\}
$$

is an open subset of $\Omega_{i}$. We have a map into the topology on $\Omega_{i}$,

$$
\lambda_{i}: X \rightarrow \mathrm{S}_{i}
$$

with the following properties:

5.1. (i) $\lambda_{i}(0)=\emptyset ; \lambda_{i}(x)=\emptyset$ if and only if $[0, x] \cap M_{i}=\{0\}$.

(ii) $\lambda_{i}(x)=\Omega_{i}$ if and only if $M_{i}$ has a one and $x$ is it.

(iii) $\lambda_{i}$ maps the elements of $M_{i}$ one-to-one onto the base sets of $S_{i}$

(iv) $x \leq y$ in $X$ implies $\lambda_{i}(x) \subseteq \lambda_{i}(y)$.
(v) If $x \wedge y$ exists in $x$ then $\lambda_{i}(x \wedge y)=\lambda_{i}(x) \cap \lambda_{i}(y)$. If $x \vee y$ exists in $X$ then $\lambda_{i}(x \vee y)=\lambda_{i}(x) \cup \lambda_{i}(y)$.

Write

$$
\mathrm{S}=\prod_{i \in I} \mathrm{~s}_{i}
$$


the elements of $S$ are all families $U=\left(U_{i}\right)_{i \in I}$ where $U_{i}$ is open in $\Omega_{i} \cdot S$ is given the weak componentwise order;

$$
U \subseteq V \text { means } U_{i} \subseteq V_{i} \text { for all } i \in I
$$

where on the right (but not on the left) $\subseteq$ denotes set inclusion. Now write

$$
\mu(x)=\left(\lambda_{i}(x)\right)_{i \in I}
$$

THEOREM 5.2. With the above notation, $(S, \subseteq)$ is a complete distributive lattice, the lattice operations being componentwise. The map

$$
\mu: X \rightarrow \mathrm{S}
$$

is a hypolattice homomorphism, sending $\emptyset$ to the zero of $S$. The map is onto $\mathrm{S}$ only if $X$ is a lattice with $I$. The map is one-to-one and isotone when restricted to $U \underline{\underline{M}}$. If $X=U \underline{M}$ then $\mu$ is a hypolattice isomorphism of $X$ onto a subhypolattice of the lattice $(S, \subseteq)$.

Proof. The homomorphism properties of $\mu$ follow immediately from 5.1. Let $x, y \in U \underline{\underline{M}}$ and $\mu(x) \leq \mu(y)$; we have $x \in M_{i}$ for some $i \in I$ and then

$$
[0, x]=[0, x] \cap M_{i} \subseteq[0, y] \cap M_{i}
$$

so $x \in[0, y]$; that is, $x \leq y$. Thus $\mu$ is isotone on $U \mathbb{M}$. The rest of the proof is straightforward. //

We remark that $\mu(X)$ may not be a convex subset of $S$.

The representation $\mu$ depends of course upon the chosen family $\underline{\underline{M}}$ of maximal sublattices. We can always find a family which covers $X$; for example the family of all maximal sublattices, for if $x \in X$ then $[0, x]$ is contained in some maximal sublattice. Thus

COROLLARY 5.3. Every Boolean hypolattice is isomorphic to a subhypolattice of a complete distributive lattice.

Now invoke the representation Theorem 4.l: to every element $U=\left(U_{i}\right)_{i \in I}$ of $\mathrm{S}$ there corresponds an open set $U_{i \in I} \sigma_{i}^{-1}\left(U_{i}\right)$ of the topological free union, in fact the map 


$$
v:\left(U_{i}\right)_{i \in I} \mapsto \bigcup_{i \in I} \sigma_{i}^{-1}\left(U_{i}\right), S \rightarrow T,
$$

where $T$ is the Stone topology on $\Psi$, is a lattice isomorphism from $(S, \subseteq)$ to $(T, \subseteq)$. Write $\xi=\nu \circ \mu$, so that, for all $x \in X$,

$$
\xi(x)=\bigcup_{i \in I} \sigma_{i}^{-1}\left\{\underline{\underline{p}} \in \Omega_{i}:[0, x] \cap M_{i} \Phi \underline{\underline{p}}\right\} \text {. }
$$

Considering only the case where $U \underline{\underline{M}}=X$, we get the main result.

THEOREM 5.4. Let $(X, \leq)$ be a Boolean hypolattice, and $\left(M_{i}\right)_{i \in I}$ any covering of $X$ by maximal sublattices. Then there exists an isomorphism

$$
\xi: X \rightarrow \mathrm{T}
$$

from $X$ onto a subhypolattice of the topology of the Stone space of the small product $K=\prod_{i \in I}^{*} M_{i}$.

We remark that $\mu$ in Theorem 5.2 and $\xi$ in Theorem 5.4 necessarily preserve complementation. Note also that they preserve de facto meets as well as de jure meets.

If $(X, \leq)$ is a Boolean lattice then we can take $\underline{\underline{M}}$ to consist of the singleton $\{X\}$, and then $\mu$ reduces to the stone representation.

We have not made reference to prime ideals of the hypolattice $X$. It is possible to define ideals in a hypolattice in several natural ways, and then prime ideals. Unfortunately these definitions seem not to lead to lemmas like the basic lemmas on which the Stone representation of Boolean lattices is based. For example, one lacks results like the following:

(1) an ideal is prime if and only if it is maximal;

(2) the collection of all prime ideals is trivially ordered by inclusion;

(3) given an ideal $A$ and an element $x$ not in $A$, there exists a prime ideal containing $A$ but not $x$.

For this reason our construction has resorted to sublattices which are Boolean lattices. 


\section{Mutuality and maximal mutual subsets}

The relation of mutuality ((3) of \$2) has some interesting properties. Call a subset $A$ of hypolattice $(X, \leq)$ mutual if $x \smile y$ for all $x, y \in A$. Every sublattice is mutual, but not every mutual subset need be a sublattice. By Zorn's lemma any given mutual subset is contained in a maximal mutual subset, which is proper when $X$ is not a lattice. For any $A \subseteq X$ write

$$
A^{\smile}=\{x \in X: x \smile a \text { for all } a \in A\} \text { when } A \neq \emptyset, \phi^{\smile}=X .
$$

Here $A^{\checkmark}$ may be empty. The map $A \mapsto A^{\checkmark}$ is a Galois connection, so is a closure operator on $P(X)$. Subset $A$ is mutual if and only if $A \subseteq A^{-}$. For all $A \subseteq X, A^{-}$is a convex subset of $X$ and hence $\left(A^{\sim}, \leq \mid A^{-}\right)$is a hypolattice. If $X$ has a zero 0 then $0 \in A^{-}$. There is a superficial resemblance between a symmetry such as $\smile$ and an orthogonality relation $\perp$, but the latter has no mutual subsets other than \{0\}, being antireflexive.

PROPOSITION 6.1. If $A$ is a mutual subset of a hypolattice $(X, \leq)$ then

$$
A \subseteq A^{\sim} \subseteq A^{\sim}
$$

and $A^{\sim-}$ is a mutual subset.

COROLLARY 6.2. If $M$ is a maximal mutual subset then

$$
M=M^{u}=M^{\top} \text {. }
$$

Let $A$ be a mutual subset. If there exists a sublattice $L$ of hypolattice $X$, with $A \subseteq L$, then there exists a smallest sublattice of $X$ containing $A$, which we denote by $A^{l}$. It is not required that $A^{l}$ be convex in $X$. In general there may exist no sublattice containing $A$; certainly there exists none if $A$ is not mutual.

If $M$ is a maximal mutual subset and if $M^{2}$ exists then

$$
M=M^{\sim}=M^{\sim}=M^{2}
$$

and $M$ is a convex sublattice of $X$.

PROPOSITION 6.3. If the hypolattice $(X, \leq)$ is coherent, then $A^{2}$ exists for every mutual subset $A$. Moreover, there exists a smallest 
convex sublattice containing $A$.

Proof. Since $X$ is coherent and $A$ is mutual, $X$ contains elements $\wedge_{i \in F} x_{i}, \bigcup_{i \in F} x_{i}$ for every finite family $\left\{x_{i}: i \in E\right\}$ of elements in A. Write

$$
B=\left\{t \in X: \underset{i \in F}{\wedge} x_{i} \leq t \leq \vee_{j \in G} y_{j}, F, G \text { finite, } x_{i}, y_{j} \in A\right\}
$$

$B$ is the smallest convex sublattice containing $A \cdot / /$

THEOREM 6.4. In any coherent hypolattice $(X, \leq)$ the maximal mutual subsets are precisely the maximal sublattices.

Proof. If $M$ is a maximal mutual subset, then it. is a sublattice, by Proposition 6.3 and the preceding remark, and if $L$ is any sublattice containing $M$ then $L$ is mutual, so $L=M$; thus $M$ is a maximal sublattice. Conversely if $K$ is any maximal sublattice and $K \subseteq A$ for a mutual subset $A$ then $A^{Z}$ exists by Proposition 6.3 and $K=A=A^{Z}$; so $K$ is maximal mutual. $/ /$

\section{References}

[1] J.C. Abbott, "Semi-boolean algebra", Mat. Vesnik 4 (19) (1967), $177-198$.

[2] M.F. Janowitz, "A note on Rickart rings and semi-Boolean algebras", Algebra Universalis 6 (1976), 9-12.

[3] John Boris Miller, "Direct summands in Z-groups", Proc. Roy. Soc. Edinburgh Sect. A 81 (1978), 175-186.

[4] Roman Sikorski, Boolean algebras, Third edition (Ergebnisse der Mathematik und ihrer Grenzgebiete, 25. Springer-Verlag, Berlin, Heidelberg, New York, 1969).

[5] M.H. Stone, "The theory of representations for Boolean algebras", Trans. Amer. Math. Soc. 40 (1936), 37-111. 
[6] M.H. Stone, "Applications of the theory of Boolean rings to general topology", Trans. Amer. Math. Soc. 41 (1937), 375-481.

Department of Mathematics, Monash University,

Clayton,

victoria 3168 . 